\title{
DEGRADATION OF POLY(ETHYLENETEREPHTALATE) WASTE TO OBTAIN OLIGOMERS USING A ZINC COMPLEX AS CATALYST
}

\author{
A. A. OVALLE-SÁNCHEZ; P. ELIZONDO-MARTÍNEZ; N. A. PÉREZ-RODRÍGUEZ; \\ E. HERNÁNDEZ-FERNÁNDEZ; M. G. SÁNCHEZ-ANGUIANO*
}

${ }^{1}$ Universidad Autónoma de Nuevo León, UANL, Facultad de Ciencias Químicas; Av. Universidad S/N Ciudad Universitaria, San Nicolás de los Garza Nuevo León CP 66451, México.

\begin{abstract}
Thermal degradation of Poly(ethylene terephthalate) (PET) bottles waste was carried out by glycolysis using triethyleneglycol (TEG) as nucleophilic agent and $N^{1}, N^{2}$-bis(2-aminobenzyl)-1,2-diaminoethane zinc(II) (ABEN), in order to test this compound as catalyst in the degradation of PET until to obtain short chains of polyester (oligomers). The depolymerization processes was tested at 180,190 and $210{ }^{\circ} \mathrm{C}$, and two molar ratios of PET:TEG (1:1.3 and 1:2.6), the amount of catalyst used was fixed at $1 \%$ in relation to PET, the best experiment of PET glycolysis was found at $190{ }^{\circ} \mathrm{C}$ during two hours in a mass relation PET:TEG 1:1.3. All products were analyzed by Fourier transform infrared spectroscopy (IR), gel permeation chromatography (GPC) and differential scanning calorimetry (DSC), the behavior of the reaction rate was studied for the best experiment by measuring the amount of degraded PET at different glycolysis time. The degradation of PET was favored with the use of ABEN, which accelerates the reaction, obtaining oligomers in the absence of an organic solvent or reaction medium, this is the most efficient catalyst found so far. The oligomers were obtained with molecular weight between 900 and $1500 \mathrm{~g} \mathrm{~mol}^{-1}$ depending on the reaction time, 60 min was the less time of the total glycolysis of PET. Also was found that the molecular weight of the oligomers increased along the glycolysis, due to the recombination with TEG and ethylene glycol produced in degradation.
\end{abstract}

Keywords: Poly(ethyleneterephthalate), degradation, catalyst, chemical recycling, glycolysis

\section{INTRODUCTION}

PET is a thermoplastic material that presents good thermal and mechanical properties; it is mainly used in the textile industry and the fabrication of bottles for carbonated drinks. The PET bottles are discarded in huge quantities, originating a serious ecologic problem because they accumulate in the environment, such as, sewer system obstruction and its accumulation in green areas prevents the natural growth of plants. Therefore, there is a growing interest in recycling PET by degradation as a partial solution to the solid waste problem, PET waste degradation consists of the decomposition by chemical agents to obtain monomers and oligomers as raw materials for the syntheses of polyesters or employed as precursors for high molecular weight polyurethanes ${ }^{1}$, polyester $^{2}$ and other specific uses ${ }^{3}$. PET can be recycled by chemical depolymerization using diols, alcohols, water and even ethanolamine $e^{4,5}$ and diethanolamine as depolymerizing agents ${ }^{6}$. Particularly, degradation by glycolysis is a process by transesterification between ester and $\mathrm{OH}$ groups in excess, to obtain bis(2hydroxyethyl terephtalate) (BHET) ${ }^{7}$. Some previous works associated to the optimal conditions to carry out the glycolysis of PET have been published, depending of the reaction conditions were obtained BHET, dimers, trimers or oligomers $^{8,9}$, the high polydispersity of these products is not suitable for use in any particular application. Glycolysis of PET is an environmentally friendly reaction because it does not generate residues, because the obtained product is used in other processes, and the molecular weight of the oligomers produced can be controlled by adjusting some reaction conditions as temperature, time, catalyst type and glycol amount. Metal acetates of zinc, lead, cobalt, cupper and manganese have been used as catalyst in the glycolysis of PET waste with ethylene glycol; the most efficient was zinc acetate ${ }^{10}$

Carta et al. reported that temperature, time, amount of glycol and the catalyst in the process of glycolysis are the most important factors that affect the degradation. They found also that an improvement on the degradation of PET could be achieved by increasing the reaction time and the amount of the glycol, producing $\mathrm{BHET}$ as the main degradation product ${ }^{11}$. When manganese acetate was used as catalyst, the glycolysis conversion rate increases significantly with temperature, time and the amount of catalyst $\mathrm{t}^{12}$; for example, at $200{ }^{\circ} \mathrm{C}$ and 3 hours of reaction were obtained oligomers with $500 \mathrm{~g} \mathrm{~mol}^{-1}{ }^{13}$. Our research group team evaluated several catalysts: chloride aluminum, lead acetate, zinc acetate, cadmium sulphate, calcium sulphate and lithium hydroxide; using triethylene glycol as nucleophile agent, $180{ }^{\circ} \mathrm{C}$ and two hours of reaction, the amount of degraded PET varied between 8 and $95 \%$ depending of the catalyst used, being lead acetate the best catalyst. However, due to the toxicity of this compound, many researchers have chosen to use mainly zinc acetate ${ }^{14,15}$.

The depolymerization of PET obtaining close to $74 \%$ BHET was reported by Pingale and Shukla with ethylene glycol (EG) and zinc chloride as catalyst, molar ratio PET:EG 1:14 and 8 hours under reflux ${ }^{16}$. This is a very long reaction time; a large amount of nucleophile agent is also required. Ghaemy et al. studied PET degradation using zinc acetate as catalysts to evaluate its influence on the degradation level at different temperatures: 135, 160, 200 and $210{ }^{\circ} \mathrm{C}$ until BHET obtained. The highest yield was obtained after 8 hours in reflux at 200 ${ }^{\circ} \mathrm{C}$ and a mole ratio of $1 / 1.2(\mathrm{PET} / \mathrm{EG})^{17}$. Glycolytic depolymerization of PET bottle waste was attempted using excess ethylene glycol in the presence of zinc acetate, sodium carbonate, sodium bicarbonate, sodium sulphate and potassium sulphate. The highest yield was achieved with zinc acetate as catalyst at $196{ }^{\circ} \mathrm{C}$, but EG:PET molar ratio between 5.4:1 and 7.6:1 ${ }^{18}$

Zinc acetate as catalyst has been widely studied in kinetics aspects on the degradation of PET process, using 1,4-butanediol and triethylene glycol, it was found that the glycolyzed product consist mainly of BHET and dimer at $190{ }^{\circ} \mathrm{C}$ and 4 hours as reaction time ${ }^{19}$. In similar researches on glycolysis of PET, the reaction has been achieved with different glycols: propylene, diethylene and triethylene, using catalysts such as manganese acetate, zinc acetate and lead acetate. Again the reaction conditions such as time, temperature and amount of glycol are high, compared with those reported in the present study ${ }^{20,21}$. The degree of degradation of PET in glycolysis depends on the type of catalyst, ionic liquids have also been used for this purpose ${ }^{22,23}$. But their synthesis is long and complicated, for this reason they are more expensive than the metallic compounds regularly used as catalysts. In addition to the conventional catalyst, some researches have been working with titanium phosphate and 1,5,7 triazabicyclo[4,4,0]dec-5-ene as catalyst in PET degradation, at 120 and 190 ${ }^{\circ} \mathrm{C}$ with glycolysis time of 6 hours, was reported that these catalysts influenced the rate of depolymerization to obtain oligomers and $78 \%$ yield of BHET $^{24}$ and hydrotalcite with ethylene glycol as solvent at 2 hours and $198{ }^{\circ} \mathrm{C}$ was complete degradation ${ }^{25}$. The combination of microwave irradiation and ionic liquid has allowed more efficient degradation, environmental friendly and economically feasible, although it is difficult to develop industrially ${ }^{26}$. Industrial interest of PET degradation is obtaining oligomers that can be used in the manufacture of other products such as polyurethanes or unsaturated polyester resins ${ }^{27,28}$.

In the most of the papers reported so far, has been used an excess of glycol or high temperature, time and catalyst to reach a high yield of degradation. The main objective in this work was used a new complex as catalyst in order to obtain oligomers, that can be used as raw material in the synthesis of unsaturated polyester resin. The $N^{l}, N^{2}$-bis(2-aminobenzyl)-1,2-diaminoethane zinc(II) complex was synthesized using the methodology reported ${ }^{29}$. Figure 1 shows the structure of the complex ABEN, it's an alternative to metallic compounds containing zinc in its structure in order to obtain mild reactions conditions for producing oligomers from PET degradation. 
<smiles>Nc1cccc(CNCCNCc2ccccc2N)c1</smiles>

Figure 1. Structure of the $N^{l}, N^{2}$-bis(2-aminobenzyl)-1,2-diaminoethane zinc(II) complex used as catalyst.

\section{EXPERIMENTAL}

\section{Materials}

The PET was obtained from post-consumed bottles that were cleaned and chopped in small pieces of $3 \mathrm{~mm}$, washed with hot water $\left(\right.$ at $\left.60{ }^{\circ} \mathrm{C}\right)$ and rinsed with acetone, dried at $70{ }^{\circ} \mathrm{C}$ during 24 hours in a vacuum oven. Triethylene glycol (99.8\%), THF (99.8\%) and acetone (99.9\%) were acquired from Aldrich and were used without further purification.

Glycolysis

$4.0 \mathrm{~g}$ of PET $(0.025 \mathrm{~mol}), 0.04 \mathrm{~g}$ of ABEN, 5.0 or $10.0 \mathrm{~g}$ of TEG $(0.033$ or $0.066 \mathrm{~mol}$ ) were mixed in a round flask of $100 \mathrm{~mL}$ equipped with a thermometer and a reflux condenser and heated at 180,190 or $210^{\circ} \mathrm{C}$ during two hours under constant stirring. After each glycolysis experiment was completed, the flask was removed from the hot plate and the product washed three times with 30 $\mathrm{mL}$ of distilled water at $60^{\circ} \mathrm{C}$, stored in a refrigerator at $4{ }^{\circ} \mathrm{C}$ for 24 hours, and filtered to extract the remaining TEG and EG produced from PET.

The remaining product was mixed with $10 \mathrm{~mL}$ of THF, the PET not degraded was collected by decantation, dried at $80^{\circ} \mathrm{C}$ for 24 hours and weighed; the conversion percentage for the glycolysis of the PET waste was defined as equation 1. The fractions of oligomers were determined as the difference in mass between initial mass of PET and the mass of the solid residue extracted with THF.

$$
\% \text { Yield }=\frac{A-B}{A} \times 100 \quad \text { Equation } 1
$$

Where $\mathrm{A}=\mathrm{g}$ of PET at the beginning of the reaction, $\mathrm{B}=\mathrm{g}$ of residual PET

The residual product obtained after decantation with THF (oligomers) were dried $80^{\circ} \mathrm{C}$ for $2 \mathrm{~h}$ and analyzed by IR using a Thermo Scientific IR200 ATR (SeZn crystal) FTIR, 32 scans per analysis, from 650 to $4000 \mathrm{~cm}^{-1}$ using a Thermo Scientific IR200 ATR (SeZn crystal) FTIR, 32 scans per analysis, from 650 to $4000 \mathrm{~cm}^{-1}$ using a Thermo Scientific IR200 ATR (ZnSe crystal), 32 scans per analysis from 650 to $4000 \mathrm{~cm}^{-1}$ using a Thermo Scientific IR200 ATR (SeZn crystal) FTIR, 32 scans per analysis, from 650 to $4000 \mathrm{~cm}-1$ using a Thermo Scientific IR200 ATR (SeZn crystal) FTIR, 32 scans per analysis, from 650 to $4000 \mathrm{~cm}^{-1}$ using a Thermo Scientific IR200 ATR (SeZn crystal) FTIR, 32 scans per analysis, from 650 to $4000 \mathrm{~cm}^{-1}$; DSC using a TA Instrument model MDSC 2920 , heating rate of $20^{\circ} \mathrm{C} / \mathrm{min}$ from 20 to $300^{\circ} \mathrm{C}$ in nitrogen atmosphere and GPC in a HP equipment (1100 model) under the following conditions: $10 \mu \mathrm{L}$ were injected from a $6 \mathrm{mg} / \mathrm{mL}$ glycolyzed PET solution into a PL Gel $10^{6} \mathrm{~A}^{\circ}$ $10 \mu \mathrm{m}(400 \mathrm{~K}-40 \mathrm{M}), 10^{5} \mathrm{~A}^{\circ} 5 \mu \mathrm{m}(40 \mathrm{~K}-4 \mathrm{M}), 10^{3} \mathrm{~A}^{\circ} 5 \mu \mathrm{m}(1 \mathrm{~K}-40 \mathrm{~K})$ column, at $40{ }^{\circ} \mathrm{C}$, THF as mobile phase, flow rate $1.0 \mathrm{~mL} / \mathrm{min}$. The products obtaine from Experiments 2 and 3 were determined the hydroxyl values according to ASTM E 1899-08. A summary of the methodology of glycolysis is shown in Scheme 1.

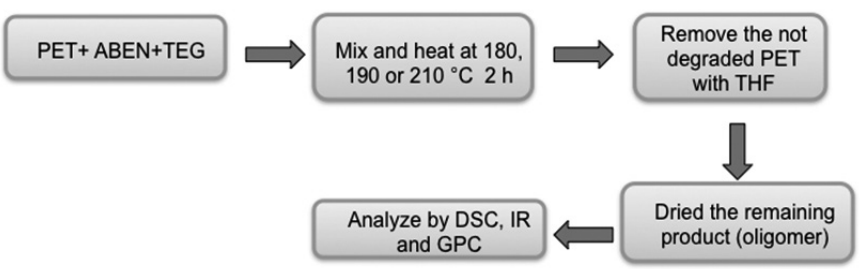

Scheme 1. Methodology used in glycolysis of PET.

Influence of time on average Mw of oligomers

From the experiment realized with 1/1.3 PET/TEG ratio and $190{ }^{\circ} \mathrm{C}$ samples were taken during the glycolysis, each 10 min during the first hour and each 20 min during the second hour, in order to studied the relationship between the glycolysis conversion of the waste PET and reaction time. The first six samples were treated with THF to separate the oligomers (degraded PET) from the PET flakes that did not react (residual PET), which was dried at $80^{\circ} \mathrm{C}$ by 6 hours and by a gravimetric method the residual PET was calculated by the equation 1 . The fraction soluble in THF was separated in a rotary evaporator; the product was dried at $80^{\circ} \mathrm{C}$ by 24 hours and weighted to obtain the oligomer mass

The aliquots taken during the second hour of reaction were not treated with THF because after $80 \mathrm{~min}$ of glycolysis the PET was totally degraded, thus these samples were treated with $30 \mathrm{~mL}$ of water at $60^{\circ} \mathrm{C}$ to extract the residual TEG produced during the degradation and separate it from the produced oligomer.

\section{RESULTS AND DISCUSSION}

Results of the degraded PET obtained in glycolysis carried out at differen temperatures and molar ratios PET/TEG are shown in Table 1 . The yields of glycolysis reactions were high in all cases, even at $180{ }^{\circ} \mathrm{C}$, the lower temperature used in this work, taking in account some references that report $74 \%$ of degradation as maximum [18]. Increasing only $10^{\circ} \mathrm{C}$ the temperature to reach $190{ }^{\circ} \mathrm{C}$ caused a $100 \%$ of degradation in the PET, thus $190{ }^{\circ} \mathrm{C}$ and a molar ratio PET/TEG 1/1.3 (Experiment 3) are the best conditions to carry out these reactions.

Table 1. Yield of PET degraded at different reaction conditions.

\begin{tabular}{|c|c|c|c|}
\hline Experiment & $\begin{array}{c}\text { Molar Ratio PET/ } \\
\text { TEG }\end{array}$ & $\begin{array}{c}\text { Temperature } \\
\left({ }^{\circ} \mathbf{C}\right)\end{array}$ & Yield (\%) \\
\hline 1 & $1 / 1.3$ & 180 & 75 \\
\hline 2 & $1 / 2.6$ & 180 & 87 \\
\hline 3 & $1 / 1.3$ & 190 & 100 \\
\hline 4 & $1 / 2.6$ & 190 & 100 \\
\hline 5 & $1 / 1.3$ & 210 & 100 \\
\hline 6 & $1 / 2.6$ & 210 & 100 \\
\hline
\end{tabular}

The IR spectrum of the product obtained from Experiment 3 is shown in Figure 2, the bands at $3420 \mathrm{~cm}^{-1}$ and $2870 \mathrm{~cm}^{-1}$, absent in the spectrum of the original PET (without degraded), are present in the product from this experiment due to the formation of chains with termination $\mathrm{OH}$ and $\mathrm{C}-\mathrm{H}$ produced during glycolysis. The functional groups shown in IR spectrum are the first indicative of polyester oligomer formation from glycolysis of PET. The other common characteristics bands of polyester resins are observed at $1714 \mathrm{~cm}^{-1}(\mathrm{C}=\mathrm{O}$ stretching), $\mathrm{C}-\mathrm{O}$ stretching at $1050 \mathrm{~cm}^{-1}, 727 \mathrm{~cm}^{-1}$ corresponding to $-\mathrm{CH}_{2}$.

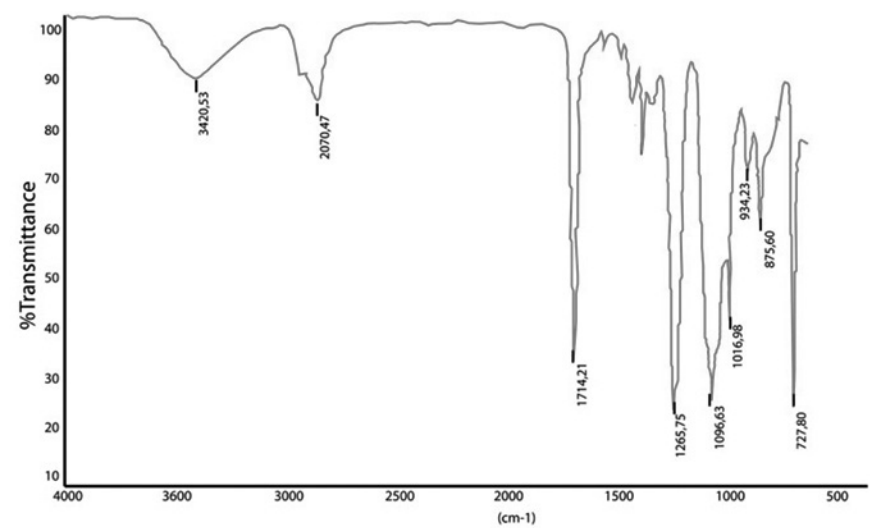

Figure 2. IR spectra of oligomer obtained from Experiment 3: PET/TEG $1 / 1.3$ ratio and $190^{\circ} \mathrm{C}$

Figure 3 shows the DSC thermogram of the product from Experiment 3 , it shows a broader peak with a principal signal at around $78^{\circ} \mathrm{C}$, this signal can be related with the fusion of a oligomers obtained and the entire peak could indicate that the oligomers consist mainly of short and mobile chains 
that apparently are modified at low temperature before melting. Since the glass transition temperature of undegraded PET is $84^{\circ} \mathrm{C}$ and melting temperature is around $260^{\circ} \mathrm{C}$

\section{Degraded PET}

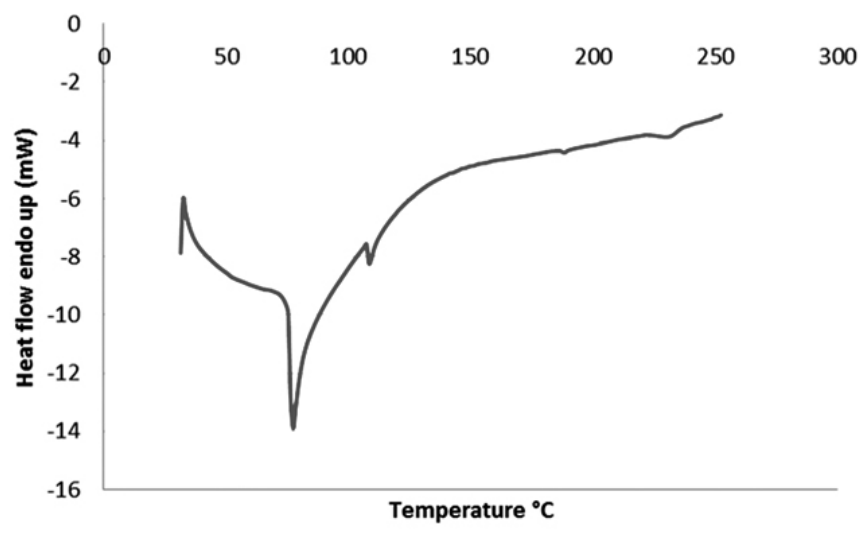

Figure 3. DSC scan of product obtained from Experiment 3 product.

The GPC results of the products obtained after glycolysis are presented in Table 2. Can be observed a lower molecular weight in the product obtained from Experiment 2 as an effect of the amount of TEG used (See Table 1), and more cleavage points caused by the $\mathrm{OH}$ groups of TEG, the $87 \%$ of the PET degraded contains smaller chains with a size uniform, therefore the polydispersity is lower.

In experiments 3 to 6 (carried at 190 and $210^{\circ} \mathrm{C}$ ) the entire PET sample was degraded demonstrating that the temperature is a more important factor than the amount of TEG used. The molecular weights were around 1,200 g/ mol indicating that the resulting oligomers are formed in average of seven repetitive units of ethylenterephtalate, this unit has a theoretical molecular weight of $196 \mathrm{~g} / \mathrm{mol}$. The hydroxyl values after glycolysis were 252.1 for the product obtained in Experiment 2 and 199.4 for the Experiment 3. The $\mathrm{OH}$ value reported of BHET is 441.5 and 251.3 for the dimer ${ }^{30}$. These results also suggest that the products obtained are mainly formed by oligomers instead pure BHET, dimers or trimers. This represents an advantage since products with a high content of $\mathrm{OH}$ groups are unsuitable for use in some applications such as waterproofing or synthesis of polyurethanes.

Table 2. Average molecular weight of the products obtained from the glycolysis.

\begin{tabular}{|c|c|c|c|c|c|}
\hline Exp & Mw & Mn & Mp & Mv & D \\
\hline 1 & 1,314 & 1,093 & 832 & 1,267 & 1.201 \\
\hline 2 & 919 & 875 & 597 & 897 & 1.050 \\
\hline 3 & 1,153 & 1,013 & 837 & 1,121 & 1.138 \\
\hline 4 & 1,162 & 1,012 & 853 & 1,126 & 1.148 \\
\hline 5 & 1,435 & 1,164 & 875 & 1,380 & 1.233 \\
\hline 6 & 1,246 & 1,047 & 874 & 1,773 & 1.190 \\
\hline
\end{tabular}

The obtained results demonstrate the effectiveness of ABEN as catalyst in glycolysis of PET. In references reported so far, it has been degraded completely PET using various catalysts; but to achieve this, high temperature is required (above $198^{\circ} \mathrm{C}$ ), solvent or excess agent. In addition, under these conditions the degradation product obtained is a mixture of BHET, terephtalic acid, dimers, trimers and oligomers ${ }^{8}$.

Complex ABEN increased the velocity of degradation of PET to get oligomers of short chains. It appears due to the catalytic activity of ABEN like as Lewis acid. The zinc interacts with the oxygen of the carbonyl group of PET, increasing the electron deficiency of carbon, this encourages the nucleophilic attack. In this sense, other research group have reported the use of $\mathrm{ZnOAc}$ like catalyst in the glycolisis of PET, revealing that the process needs higher temperature, reaction time or excess glycol that when ABEN is used ${ }^{7-9}$. This could be explained because the zinc in ABEN makes the carbonyl carbon more electrophilic and therefore more susceptible to attack by the nucleophile agent The mechanism proposed is showed in the Scheme 2.

Influence of time on average Mw of oligomers

A detailed study of the reaction in Experiment 3 was carried out to establish the rate of PET degradation under the glycolysis conditions used. Samples were taken at different reaction times and each sample was analyzed to evaluate de amount of degraded PET and its molecular weight. Table 3 shows the results of this study, at 60 min of reaction the PET was totally degraded, and the oligomer resulted in an average molecular weight about 1467. After two hours of reaction, the molecular weight $(1544 \mathrm{~g} / \mathrm{mol})$ indicates that oligomers are formed by seven ethylenterephtalate units on average.

Table 3. Average molecular weight along glycolysis time of Experiment

\begin{tabular}{|c|c|c|c|c|}
\hline Time (min) & $\begin{array}{c}\text { PET Degraded } \\
\mathbf{( \% )}\end{array}$ & Mw & Mn & D \\
\hline 10 & 21 & 1199 & 921 & 1.302 \\
\hline 20 & 40 & 1218 & 1010 & 1.206 \\
\hline 30 & 82 & 1227 & 1009 & 1.216 \\
\hline 40 & 87 & 1260 & 1015 & 1.241 \\
\hline 50 & 97 & 1354 & 1078 & 1.256 \\
\hline 60 & 100 & 1467 & 1114 & 1.318 \\
\hline 80 & 100 & 1295 & 1060 & 1.222 \\
\hline 100 & 100 & 1385 & 1082 & 1.280 \\
\hline 120 & 100 & 1544 & 1147 & 1.346 \\
\hline
\end{tabular}

Figure 4 shows the data presented in Table 3 . The cleavage of PET chain is a random phenomenon, the amount of TEG available at the beginning suffered a drastic diminution because it is grafted in the oligomers produced during the reaction, but the liberation of ethylene glycol from the PET originate a recombination reaction of these oligomers, thus, the glycolysis is a reversible process that can originate a slightly increase in the molecular weight, this behavior has already been reported ${ }^{31}$.

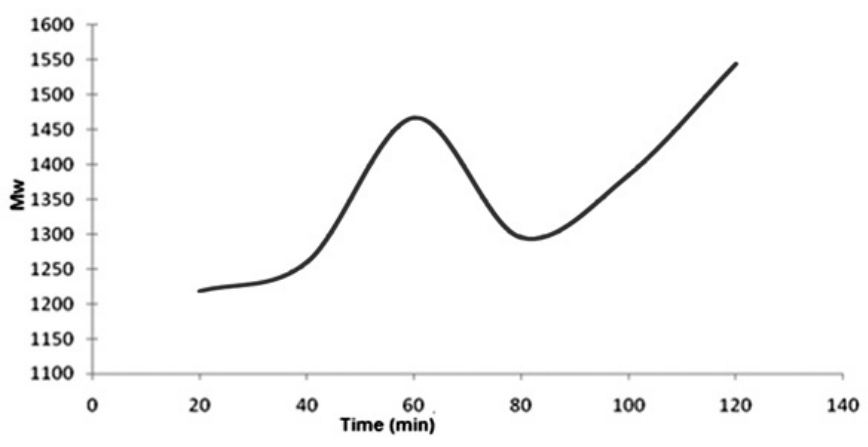

Figure 4. Molecular weight distribution of oligomers resulted from PET degradation along glycolysis time in Experiment 3.

Figure 5 indicates the relationship between the degraded PET and the glycolysis time. The amount of degraded PET increase along the glycolysis time approached a steady conversion after 60 minutes, after that time the PET is completely degraded.

In the Experiment 3 the signal intensity at $3400 \mathrm{~cm}^{-1}$ corresponding to $\mathrm{OH}$ group was analyzed by IR, in samples acquired each 20 min during the reaction. Figure 6 shows the spectra for each sample. The intensity of $\mathrm{OH}$ signal increases with the reaction time until it reaches a maximum at $80 \mathrm{~min}$, which confirms the formation of oligomers with $\mathrm{OH}$ termination during the degradation. A decrease in the signal can be observed in 100 and 120 min spectra probably because the $\mathrm{OH}$ groups are occupied in the recombination process. 

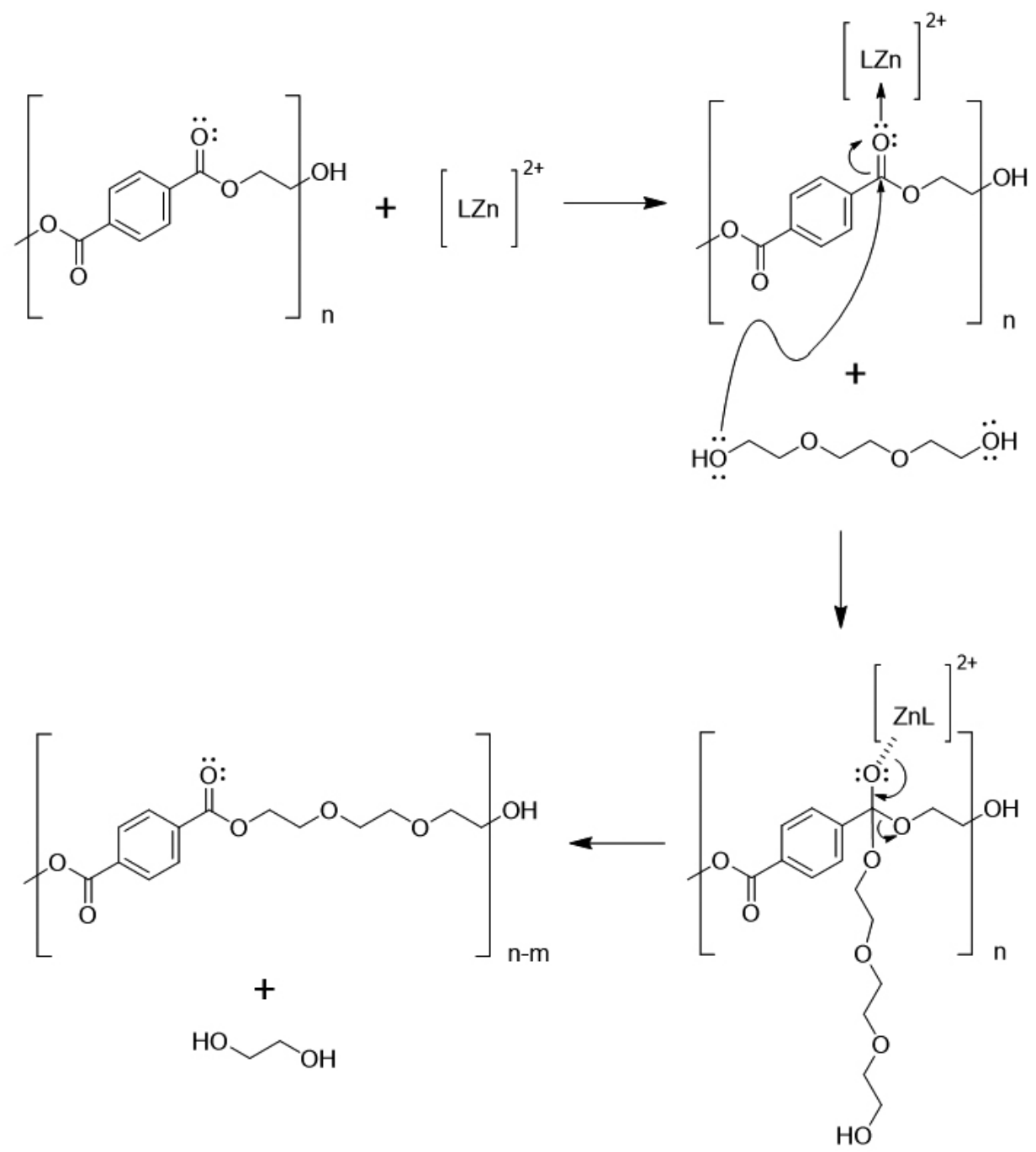

Scheme 2. Proposed mechanism of the glycolysis of PET catalyzed by ABEN.

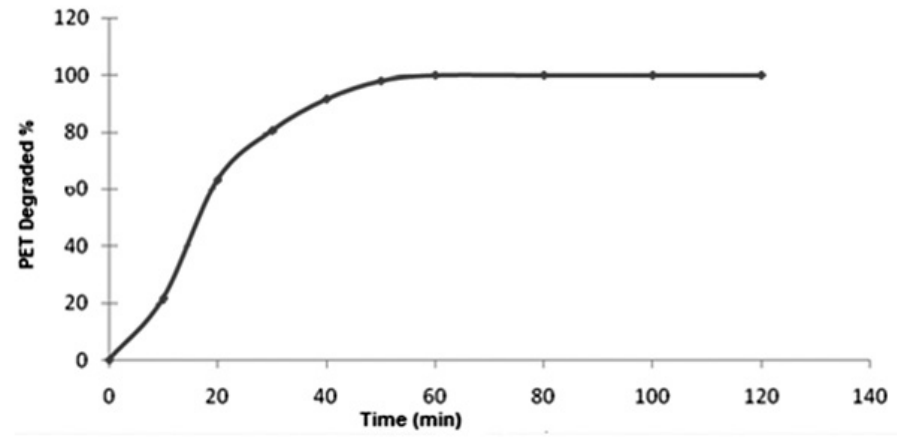

Figure 5. Relationship between PET degraded and the glycolysis time in Experiment 3.

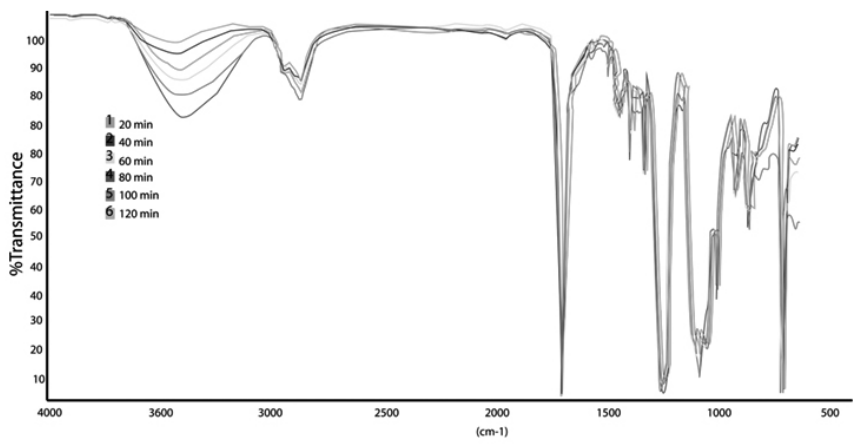

Figure 6. The IR spectroscopy of the product from Experiment 3 along reaction time. 


\section{CONCLUSIONS}

The degradation of PET is favored with the use of $N^{1}, N^{2}$-bis-(2aminobenzyl)-1,2-diaminoethane zinc(II) as catalyst, which accelerates the reaction obtaining oligomers with PET/TEG molar ratio of $1: 1.3$, reaction temperature of $190^{\circ} \mathrm{C}$ for two hours and in the absence of an organic solvent as reaction medium.

According the presented results it can be concluded that 60 minutes is the optimal time to degrade PET under the reaction condition here showed. At this time the PET is completely degraded. During the glycolysis of PET are obtained oligomers with molecular weight depends on the time of reaction, due to the recombination of the oligomers with ethylene glycol produced in degradation. The amount of TEG is an important factor, since can be modulated to control the molecular weight of the oligomers obtained. The product obtained to the degrader PET under the conditions reported in this work, can be used as raw materials in the formulation of adhesive and paints, contributing in this way to the PET recycling.

\section{REFERENCES}

1.- S.M. Cakić, I.S. Ristić, M. M-Cincovik, N.C. Nikolić, O.Z. Ilić, D.T. Stojiljković, J.K. B-Simendić, Prog. Org. Coat. 74, 124, (2012)

2.- C. Gioia, M. Vannini, P. Marchese, A. Minesso, R. Cavalieri, M. Colonna, A. Celli, Green Chem. 16, 1808, (2014)

3.- A. El-Mejjatti, T. Harit, A. Riahi, R. Khiari, I. Bouabdallah, F. Malek, Polymer Letters 8, 544, (2014)

4.- A.P. More, R.A. Kute, Iran. Polym. J. 23, 59, (2014)

5.- M.E. Tawfik, S.B. Eskander, Polym. Degrad. Stabil. 95, 187, (2010)

6.- R.M. Musale, S.R. Shukla, Int. J. Plast. Technol. 20, 106, (2016)

7.- A. Aguado, L. Martínez, L. Becerra, M. Arieta-Araunabeña, S. Arnaiz, A. Asueta, I. Robertson, J. Mater. Cycles Waste 16, 201, (2014)

8.- M.F.A. Viante, C.S. Nunes, E.C. Muniz, M.L. Felsner, C.A.P. Almeida, Polym. Int. 65, 1024, (2016)

9.- A.M. Al-Sabagh, F.Z. Yehia, Gh. Eshaq, A.M. Rabie, A.E. ElMetwally, Egyptian J. Of Petroleum. 25, 53, (2016)

10.- A. Ptičck, A. Fijačko, Z. Hrnjak-Murgič, Chem. Biochem. Eng. 27, 65, (2013)
11- D. Carta, G. Cao, C. D`Angeli, Environ. Sci. Pollut. R. 10, 390, (2003)

12.- Ch. H. Chen, J. Appl. Polym. Sci. 87, 2004, (2003)

13.- B. Geyer, G. Lorenz, A. Kandelbauer, Express Polym. Lett. 10, 559, (2016)

14.- M.E. Viana, A. Riul, G.M. Carvalho, A.F. Rubira, E.C. Muniz, Chem. Eng. J. 173, 210, (2011)

15.- N. George, T. Kurian, Ind. Eng. Chem. Res. 53, 14185, (2014)

16.- N.D. Pingale, V.. Palekar, S.R. Shukla, J. Appl. Polym. Sci. 115, 249, (2010)

17.- M. Ghaemy, F. Behzadi, Iran. Polym. J. 11, 77, (2002)

18.- R. López-Fonseca, I. Duque-Ingunza, B. Rivas, S. Arnaiz, J. GutierrezOrtiz, Polym. Degrad. Stab. 95, 1022, (2010)

19.- M.E. Viana, A. Riul, G.M. Carvalho, A.F. Rubira, E.C. Muñiz, Chem. Eng. J. 173, 210, (2011)

20.- M.Y. Abdelaal, T.R. Sobahi, M.S. Makki, Constr. Build. Mater. 25, 3267, (2011)

21.- S.R. Shukla, A.M. Harad, J. Appl. Polym, Sci. 97, 513, (2005)

22.- H. Wang, Y. Liu, Z. Li, X. Zhang, S. Zhang, Y. Zhang, Eur. Polym. J. 45, $1535,(2009)$

23.- M. Zhu, S. Li, Z. Li, X. Lu, S. Zhang, Chem. Eng. J. 185, 168, (2012)

24.- K. Fukushima, O. Coulembier, J.M. Lecuyer, H.A. Almegren, A.M. Alabdulrahman, F.D. Alsewailem, M.A. Mcneil, P. Dubois, R.M. Waymouth, H.W. Horn, J.E. Rice, J.L. Hedrick, J. Polym. Sci. Part A: Polym. Chem. 49, 1273, (2011)

25.- V. Sharma, P. Shrivastava, D.D. Agarwal, J. Polym. Res. 22, 241, (2015)

26.- M.A. Alnaqbi, M.A. Mohsin, R.M. Busheer, Y. Haik, J. Appl. Polym. Sci. Doi: 10.1002/APP.41666, (2015)

27.- M.Y. Abdelaal, T.R. Sobahi, M.S.I. Makki, Constr. Build. Mater. 25, 3267, (2011)

28.- A.R. Zahedi, M. Rafizadeh, F.A. Taromi, J. Thermoplast. Compos. Mat. 27,1256, (2014)

29.- P. Elizondo, B. Nájera, N.A. Pérez, L. Hinojosa, M.I. Gómez J. Serb. Chem. Soc. 78, 591, (2013)

30.- V. Pimpa, R. Sirisook, S. Chuayjuljit, J. Appl. Polym. Sci. 88, 788, (2003)

31.- R.M.K. Prado, Ch.R. Nascimento, Ch. Azuma, M.L. Dias, Prog. Rubber. Plast. Re. 24, 183, (2008) 